\title{
Cochlear Implantation in Children with Cardiac Conditions: Experience in a Tertiary ENT Centre in India
}

Dr. Kiran Natarajan, Consultant ENT Surgeon, Madras ENT Research Foundation, Chennai, India

Dr. M. Jagannath, Senior Consultant Anesthetist, Madras ENT Research Foundation, Chennai, India

Dr. Madan Mohan, Consultant Cardiologist, Madras ENT Research Foundation, Chennai, India

Prof. Mohan Kameswaran, Senior Consultant ENT Surgeon, Madras ENT Research Foundation, Chennai, India

\section{Introduction}

Profound deafness may be associated with cardiac conditions which may influence cochlear implantation $(\mathrm{Cl})$.

Objectives

- To analyze the various cardiac conditions encountered in 1000 cochlear implantees from 1997 till date

- To develop a protocol for managing cardiac issues in $\mathrm{Cl}$

Materials and Methods

- History and ENT examination

- Audiology and Electrophysiology

- CT and MRI of temporal bone, brain

- Consults - Cardiology, Pediatric, Ophthalmology, Anaesthesiology, Clinical Psychology, Occupational Therapy

Observation and Results

- Cardiac conditions - in 90 profoundly deaf candidates (9\%)

\section{Categorization of cardiac conditions}

\begin{tabular}{|c|l|c|c|}
\hline Groups & \multicolumn{1}{|c|}{ Features } & $\begin{array}{c}\text { Numbers } \\
\mathbf{( n = 9 0 )}\end{array}$ & $\begin{array}{c}\text { Percentage } \\
\mathbf{( 9 \% )}\end{array}$ \\
\hline A & Isolated Patent Ductus Arteriosus & 16 & $1.6 \%$ \\
\hline B & $\begin{array}{l}\text { Syndromes and other anomalies } \\
\text { associated with PDA }\end{array}$ & 24 & $2.4 \%$ \\
\hline C & Syndromes without PDA association & 50 & $5 \%$ \\
\hline
\end{tabular}

Group A - 16 patients - diagnosed with PDA during pre-op evaluation for $\mathrm{Cl}$

Group B

\begin{tabular}{l|c|c}
\multicolumn{1}{c|}{ Patent Ductus Arteriosus (PDA) with } & Numbers & Percentage \\
\hline Rubella & 10 & $1 \%$ \\
\hline Pulmonary stenosis & 4 & $0.4 \%$ \\
\hline Pulmonary stenosis and Rubella & 5 & $0.5 \%$ \\
\hline Pulmonary stenosis, Rubella and Patent & 2 & $0.2 \%$ \\
\hline Foramen Ovale & 1 & $0.1 \%$ \\
\hline Goldenhar syndrome & 2 & $0.2 \%$
\end{tabular}

\section{Group C}

- Jervell Lange Nielsen (JLN) Syndrome - 50 patients (5\%)

- Long QTc in ECG - greater than 440 msec

- Tachyarrhythmias, including ventricular tachycardia, Torsade de pointes, ventricular fibrillation

- Syncopal attacks, sudden death

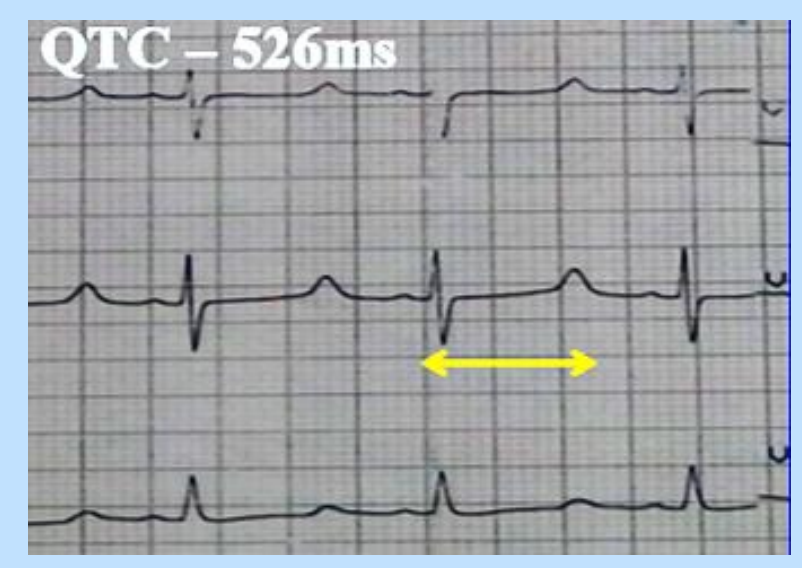

ECG - Long QTC
Management protocol

Pre-operative

- Premedication

- Infective endocarditis prophylaxis

- Air filter in the IV line to prevent air embolism

Intra- \& Post-operative (Group A \& B)

- $\mathrm{Cl}$ - with Med EL pulsar device

- Balanced anesthesia

- Systemic vasoconstrictors avoided

- Normoxia, normocarbia, normothermia, normotension

- Fluid overload avoided

- Diuretics - SOS

- Awake Extubation

- Post-op - Nasal $\mathrm{O}_{2}$ for 6 hours

Pre-operative (Group C)

- Long QTc interval - corrected by pre-op beta blocker

- Maintainance of normal serum $\mathrm{K}+, \mathrm{Ca} 2+$ and Mg2+

Intra-operative (Group C)

- Propofol for induction

- Inhalational agents avoided

- Maintenance with Propofol, nitrous oxide and oxygen

- Intermittent fentanyl, atracurium,

- IV magnesium sulfate, Antiarrhythmic drugs e.g. Beta blockers - SOS

- Electrocautery - avoided

- Drugs that prolong QT interval - avoided

- Pacing - SOS

- Defibrillatlon (1J / Kg) - SOS

- Avoiding Neostigmine reversal

- Spontaneous recovery

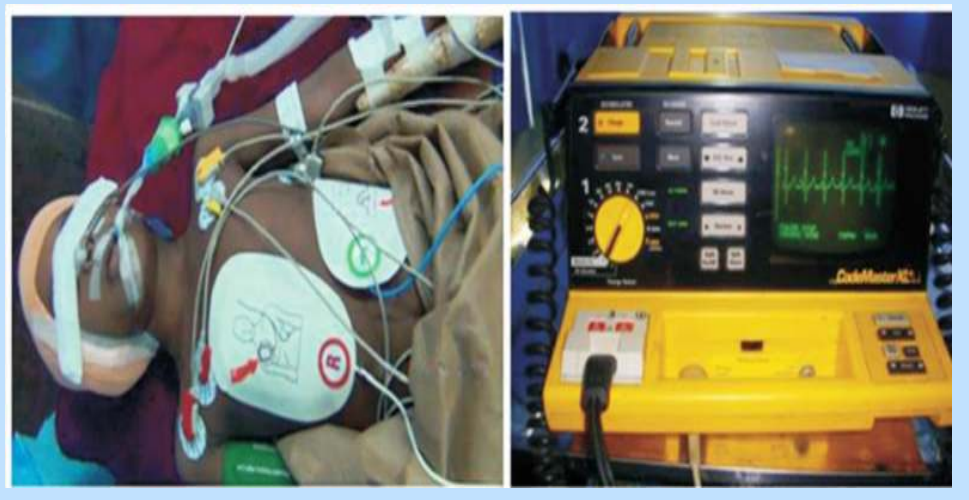

Defibrillator on stand-by

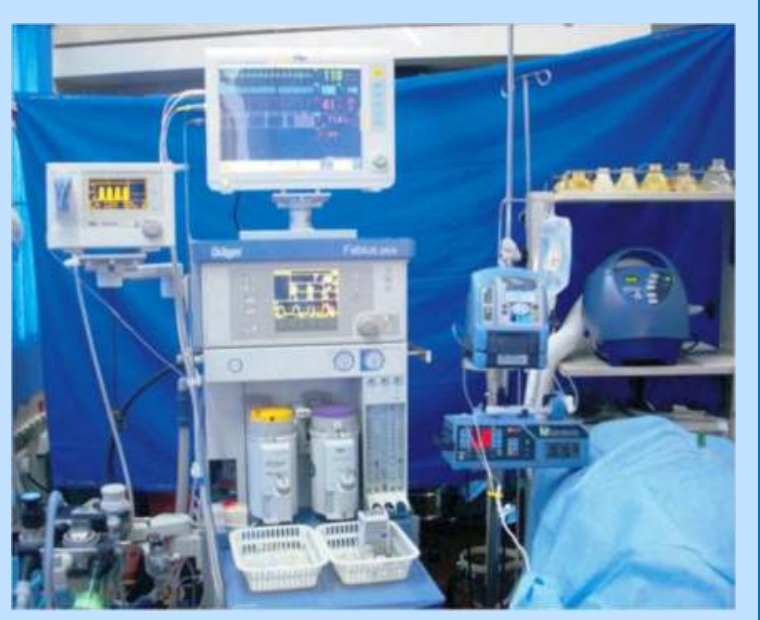

Intra-op monitoring
Post-operative (Group C)

- Intensive care unit monitoring - continuous ECG, SpO2

- Good analgesia

- Post-op Beta blockers - if QTc > $480 \mathrm{msec}$

- Demand Pacing - SOS

- AVOID DRUGS THAT PROLONG QT INTERVAL

\section{Conclusion}

- Meticulous investigative protocol in children presenting with hearing loss is mandatory.

- Issues related to pre-, intra- and post-operative care have to be appropriately addressed in children with cardiac conditions undergoing cochlear implantation.

\section{References}

1.Taussig HB. Patent Ductus Arteriosus and Loss of Hearing. Ann Intern Med. 1968;69:167.

2.De Santis M, Cavaliere AF, Straface G, Caruso A. Rubella infection in pregnancy. Reprod. Toxicol. 2006;21(4):390-8.

3.Green JD, Schuh MJ, Maddern BR, Haymond J, Helffrich RA.

Cochlear implantation in Jervell and Lange-Nielsen syndrome. Ann Otol Rhinol Laryngol Suppl. 2000;185:27-8.

4.Senthil Vadivu A, Kameswaran M. Int J Ped Otorhinolaryngol. 2013;77:1303-1307.

5.Baidya DK, Dehran M. Anesthesia for cochlear implant. Trends Anaesth Crit Care. 2011;1:90-4. 